\title{
Propuesta constructivista para la enseñanza del campo eléctrico
}

\author{
Construction proposal for the teaching of the electric field
}

DOI: https://doi.org/10.33262/rmc.v6i1.1068

\author{
Octaviano Cerriteño Rodríguez ${ }^{1}$ \\ Instituto Tecnológico de Querétaro, México \\ https://orcid.org/0000-0001-8762-3961 \\ profecerri@yahoo.com.mx
}

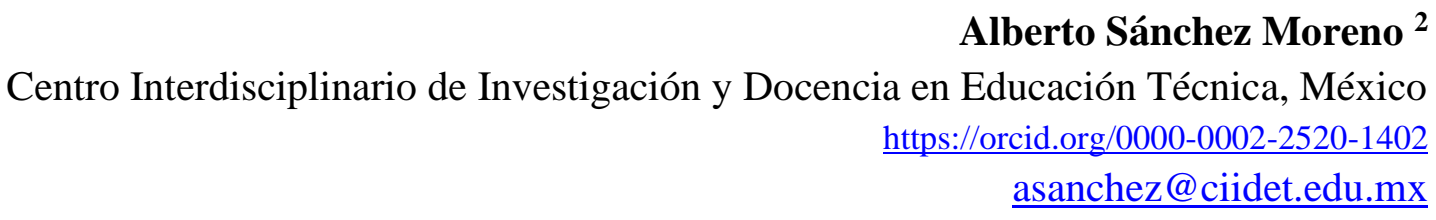

José Luis Álvarez López ${ }^{3}$

Centro Interdisciplinario de Investigación y Docencia en Educación Técnica, México

https://orcid.org/0000-0002-4163-1568

jalvarez@ ciidet.edu.mx

José Ricardo Aguilera Terrarts 4

Centro Interdisciplinario de Investigación y Docencia en Educación Técnica, México

https://orcid.org/0000-0001-5086-3259

raguilera@ ciidet.edu.mx

\section{RESUMEN}

Se presenta una propuesta didáctica para enseñar el concepto de campo eléctrico en ingeniería. Se sigue un planteamiento teórico basado en el enfoque constructivista partiendo de los conocimientos previos de los alumnos, identificados mediante un diagnóstico inicial y un mapa cognitivo, y se propone emplear una serie de experimentos sencillos de fenómenos eléctricos para que ellos reflexionen sobre las interacciones observadas. Con la propuesta también se aspira a que los estudiantes den una explicación del porqué de los fenómenos a través de preguntas dirigidas que inducen a considerar la posibilidad de incluir conceptos científicos en sus explicaciones. Lo anterior permitirá que los estudiantes adquieran un aprendizaje significativo de los conceptos estudiados, haciéndolos propios e integrándolos a sus conocimientos existentes.

PALABRAS CLAVE: Enseñanza y aprendizaje, Proceso cognitivo, Dificultad de aprendizaje. 


\section{ABSTRACT}

A didactic proposal is presented to teach the concept of electric field in engineering. A theoretical approach based on constructivism approach is followed, based on the students' previous knowledge, identified through an initial diagnosis and a cognitive map, and it is proposed to use a series of simple experiments of electrical phenomena so that they reflect on the observed interactions. The proposal also aims for students to give an explanation of the why of the phenomena, through directed questions that induce them to consider the possibility of including scientific concepts in their explanations. This will allow students to acquire a meaningful learning of concepts studied, making them their own and integrating them with their existing knowledge.

KEYWORDS: Teaching and learning, Cognitive process, Learning Disability.

\section{INTRODUCCIÓN}

Algunos investigadores en educación de las ciencias como Moreira et al. (2002) han indicado que la forma en que los alumnos aprenden un concepto es, en general, diferente a la manera como se intenta enseñar, tanto en el campo de la ciencia como en otras disciplinas. En un campo científico muy específico, en la enseñanza y aprendizaje de los fenómenos eléctricos, los conceptos no son bien afianzados por los alumnos, resultando la mayoría de las veces en un aprendizaje memorístico y rutinario. Estas dificultades no son exclusivas de la física, ya que también son referidas a las matemáticas, ciencias de la Tierra, química, entre otras. Es decir, la problemática es observable en el área de las ciencias básicas. Entre los diversos factores que pueden intervenir se encuentra la preparación deficiente con la que llegan los alumnos a los cursos de Física, así como la falta de motivación para el aprendizaje del conocimiento científico. Estas dificultades, y su compleja solución, han impedido alcanzar los resultados de aprendizaje esperados (Pozo, 1997; Hernández, 2000).

En México existe una problemática similar. Si bien ésta no ha sido ampliamente documentada, hay algunos trabajos de investigación que indican que existen resultados desfavorables para el proceso enseñanza aprendizaje, como los relacionados con el problema de la falta de comprensión de conceptos de la electrostática, líneas de fuerza y campo eléctrico (Mora y Sandoval, 2009).

En los Institutos Tecnológicos pertenecientes al Tecnológico Nacional de México (TecNM), órgano desconcentrado de la Secretaría de Educación Pública, se cursan $\underline{18}$ carreras y atiende a una población escolar de más de 600 mil estudiantes en licenciatura y posgrado en todo el territorio nacional, incluida la Ciudad de México (para mayor referencia consultar https://www.tecnm.mx/). La formación del estudiante está orientada principalmente al conocimiento y uso de la tecnología lo que implica la necesidad de una sólida preparación en las ciencias básicas, principalmente en física y matemáticas. De esta manera, los estudiantes cursan alrededor de 15 asignaturas basadas en estas áreas del conocimiento; sin embargo, se reportan serias dificultades en su enseñanza y aprendizaje provocando así que el estudiante promedio, en el mejor de los casos, requiera de un tiempo mayor al programado para terminar sus estudios o, en el peor de los casos, 
abandone su formación profesional al causar baja de la institución por reprobar estas asignaturas (Jiménez, 2011).

Esta realidad del TecNM motiva a investigar tal problemática a través de la pregunta ¿qué alternativas se pueden proponer para mejorar la enseñanza y el aprendizaje de las Ciencias Básicas y reducir así el índice de reprobación en los Institutos Tecnológicos del TecNM? En este trabajo intentaremos dar una respuesta a esta pregunta a través de una propuesta de enseñanza de la física que ejemplificaremos con el tema de campo eléctrico.

La dificultad en la enseñanza de la electricidad se divide en dos aspectos: a) la abstracción y el lenguaje de su expresión y b) las matemáticas. Los conceptos físicos como carga, interacción a distancia y campo eléctrico son abstractos (en el sentido de una representación simbólica construida para modelar y manipular objetos e ideas en un espacio matemático) y existen muy pocos ejemplos de fenómenos concretos o cotidianos que puedan utilizarse como analogías para facilitar su comprensión; además, tal comprensión se dificulta en tanto que se trata de objetos que no están necesariamente en contacto uno con respecto a otro, pero con una influencia recíproca ejercida (Llancaqueo, 2006).

El lenguaje natural de la física es la matemática, ya que es necesario poseer y aplicar los conocimientos elementales del álgebra vectorial para manipular los conceptos físicos del tema. Por tal motivo, el desconocimiento del lenguaje matemático, por parte de los estudiantes, puede considerarse como la primera dificultad en el proceso de aprendizaje, porque a nivel bachillerato no se enseñan vectores — salvo en muy escasas excepcionesy en las carreras de ingeniería, donde se requiere mayor profundidad conceptual, es necesario aplicar tanto el álgebra vectorial como tener un dominio de geometría elemental, de trigonometría, y ciertos conceptos de geometría analítica que permitan la comprensión de la interacción eléctrica. Por lo tanto, los alumnos que cursan la materia de Electromagnetismo en carreras como Ingeniería Eléctrica, Ingeniería Electrónica y Mecatrónica, tienen una doble dificultad que enfrentar: por un lado, los conocimientos matemáticos a manejar y, por otro, las nociones físicas previas que requieren comprender. Consideramos que el proceso de enseñanza está completo cuando el estudiante ha aprendido y esto se da cuando ya ha logrado formar nuevos esquemas e integrarlos a su estructura cognitiva, debiendo en algunos casos de modificarla y romper con ideas erróneas que tenía al iniciar un curso. Tal creación de estructuras de conocimiento mediante la relación sustantiva entre nueva información e ideas previas lo lleva a alcanzar un aprendizaje significativo que supera a la simple memorización (Díaz-Barriga y Hernández, 2010); además, esta forma de aprendizaje desarrolla la adquisición de habilidades y destrezas que les permitirá aplicar esos conocimientos a diversos contextos de su desarrollo profesional.

Esto se puede lograr mediante la aplicación de diversos elementos teóricos del aprendizaje, tales como esquema, estructura, aprendizaje significativo, andamiaje, etc., al ámbito de las ciencias básicas, permitiendo relacionar el conocimiento científico con el entorno psicopedagógico, lo que significaría la exploración en el ámbito interdisciplinario y quizás una transversalidad en las mismas (transdisciplinario) que supere la fragmentación del conocimiento de las ciencias particulares, así como su especialización. 
Ante la falta de estudios sistemáticos de la enseñanza y aprendizaje en ingeniería (la mayoría se orienta hacia trabajo experimental y uso de analogías), resulta indispensable profundizar en el conocimiento del problema que permita generar propuestas didácticas, y que contribuyan en lo general al mejoramiento de la calidad educativa en el sistema de educación superior, y en lo particular a la enseñanza de la asignatura de Electromagnetismo.

El concepto de campo eléctrico, así como sus propiedades y cuantificación, son aspectos medulares en la enseñanza de la electricidad que, desafortunadamente, han tenido dificultad en ser aprendidos por los estudiantes. En las siguientes secciones se presenta una alternativa de solución a esta problemática.

\section{DESARROLLO}

La propuesta educativa que aquí se presenta es un modelo didáctico constructivista o constructor (Campelo, 2003; Ruiz, 2007; Flórez, 2005; Hernández, 2000; Díaz-Barriga y Hernández, 2010), en la cual se considera que el alumno es un sujeto con personalidad propia que aprende significativamente de acuerdo a sus habilidades cognitivas, y que por lo tanto tiene capacidad para aprender y solucionar problemas, así como desarrollarse usando nuevos aprendizajes y habilidades estratégicas (Carretero, 2009).

El modelo que se ha seleccionado es por su génesis una derivación de la teoría cognitiva de la equilibración de Piaget y de la teoría del aprendizaje significativo de Ausubel (Rivera, 2004), aunque para el caso de la enseñanza de las ciencias se le suele denominar de investigación (Mayorga y Madrid, 2010; Ruiz, 2007). El modelo didáctico se ha adaptado a partir de diferentes teorías que tienen como factor común que el conocimiento humano es construido activamente por las personas, de tal manera que en el aula se integren pensamientos, emociones y acciones.

La construcción del conocimiento que elabora cada persona es ante todo un proceso racional, y en consecuencia es susceptible de aplicar instrucción educativa. Es de suponer que existen factores motivacionales y emocionales, que no son considerados en este artículo, que afectan al proceso de aprendizaje y que complican el proceso constructivo. Por lo tanto, sólo tomaremos algunos puntos de vista de la psicología educativa respecto a la motivación en el aula, tratando de dar una orientación a la didáctica que permita en los alumnos la construcción de ideas y conceptos.

Un concepto fundamental que no debe soslayarse es que la construcción del conocimiento por parte de la persona que aprende mantiene tres condiciones necesarias para lograr el aprendizaje significativo: 1) que la nueva información sea potencialmente significativa y sea clara, organizada y consistente; 2) que los alumnos dispongan de conocimientos previos que les permitan asimilar y elaborar los nuevos conocimientos; 3) finalmente, que tengan la disposición -y quizá las herramientas cognitivas - para aprender significativamente y no sólo de manera memorística (Campelo, 2003; Moreira, 1977). El segundo punto hace énfasis en lo que el alumno ya sabe, esto es, en los conocimientos previos presentes en su estructura cognitiva (Campelo, 2003). Los nuevos conocimientos deberán ser una alternativa viable y potencialmente relevante para que el estudiante verdaderamente perciba y sienta inquietud por los cambios del conjunto de ideas que 
según él conocía; ello será el motor para llevarlo a la acción y finalmente a una real reconstrucción de su conocimiento en su estructura cognitiva.

La teoría de la Equilibración de Piaget nos dice que debe generarse un desequilibrio cognitivo en el aprendiz, quien buscará nuevamente el equilibrio de su estructura conceptual y psicológica a través de los procesos de organización y adaptación, de modo que el individuo aprende los nuevos conocimientos (esquemas) y los adapta a su estructura cognitiva, y que a su vez tales conocimientos modifican al alumno en referencia al cambio cognitivo (Saldarriaga-Zambrano, et al., 2016). Sin embargo, ese desequilibrio inicial suele ser engañoso pues, en la práctica docente común, el estudiante ha construido un conocimiento cotidiano aprendido a lo largo de su vida y por medio del cual ha podido explicar y dar solución a los problemas con los que se ha enfrentado, aun cuando haya partido de ideas erróneas. Aunado a ello, los profesores intentan enseñar la ciencia como un conjunto de conocimientos verdaderos, los presentan de manera desvinculada de la vida cotidiana de su auditorio, lo que no favorece el aprendizaje (Flórez, 2005).

Lo que sucede es que las explicaciones científicas no llegan a surtir el impacto necesario, dado que éstas no se contrastan con las ideas simplistas basadas en la mera experiencia, sino que el conocimiento escolar y el cotidiano marchan en paralelo y nunca se confrontan (Flórez, 2005). Esta circunstancia genera en muchos estudiantes, factores de resistencia para alcanzar un aprendizaje significativo, particularmente de los nuevos conceptos científicos, que no se relacionan con la experiencia cotidiana y los conceptos generados de ella.

Cabe destacar que el aprendizaje no se restringe al trabajo del docente y del estudiante, sino también del trabajo que realiza el grupo como colectivo, esto es, el intercambio de ideas entre los compañeros, trabajo colaborativo, la ayuda para estudiar y comprender conceptos, etc., todo dentro del ámbito escolar. Para el modelo pedagógico constructivista, es clave la consideración de la denominada zona de desarrollo próximo propuesta por Vygotsky, la cual se refiere al potencial de aprendizaje que los alumnos pueden lograr con la ayuda de un compañero, un adulto o un maestro, dentro de su entorno escolar o social (Carretero, 2009).

Aunque Vygotsky no planteó teorías constructivistas, con la idea de que cada persona pudiera construir su propio conocimiento, se considera que la interacción social con los compañeros y con el profesor es necesaria para que cada alumno cree nuevo conocimiento (Díaz-Barriga y Hernández, 2010).

No hay una sola teoría que fundamente jerárquicamente al constructivismo, más bien, se trata de diversos modelos que confluyen a un conjunto de principios y conceptos explicativos de naturaleza constructivista seleccionados de la teoría que los origina (Coll, 2001). Además, es necesario considerar el entorno escolar para afianzar las relaciones entre el aprendizaje y desarrollo de los alumnos, así como de la participación de los docentes, por citar sólo las que se considerarán en este artículo. Otras definiciones de constructivismo son las dadas por Carretero (2009) o Díaz-Barriga y Hernández (2010) resumidas en la Tabla 1. 
Tabla 1.

Concepciones del constructivismo.

\begin{tabular}{c|l}
\hline \multicolumn{2}{c}{ Carretero } \\
\hline El constructivismo se debate ante tres puntos de vista: \\
\hline 1 & El aprendizaje es una actividad solitaria. \\
2 & Con amigos se aprende mejor. \\
3 & Sin amigos no se puede aprender. \\
\hline
\end{tabular}

Díaz-Barriga y Hernández

El constructivismo tiene las siguientes ideas comunes:

$1 \quad$ El aprendizaje es un proceso que se da en el cerebro de cada persona, de modo que el aprendiz construye (y reconstruye) su propio conocimiento.

$2 \quad$ El aprendizaje será más eficaz en la medida que tenga mayor interacción con los demás.

$3 \quad$ El docente es mediador del aprendizaje, se convierte en guía y orientador de la construcción del aprendizaje de cada aprendiz.

Fuente: Carretero (2009, p. 34-35); Díaz-Barriga y Hernández (2010, p. 27-28)

En cuanto al modelo didáctico constructivista, es necesario señalar que las personas no aprenden directamente los conceptos enseñados por los profesores, sino a través de representaciones mentales que se van construyendo en cada persona (Moreira et al., 2002). Por esta razón, los alumnos no son pasivos recipientes de información, sino activos constructores de su conocimiento. Según estos autores, aprender ciencias significativamente implica que los aprendices "sean capaces de recrear las teorías físicas en sistemas de representación internos de conceptos relacionados" (Moreira et al., 2002, p. 39). Al ser el docente un mediador entre el aprendizaje constructivista de los alumnos y el conocimiento como un cuerpo conceptual establecido por los científicos especialistas en el tema, sus métodos y estrategias de enseñanza resultan fundamentales para establecer una buena relación con sus alumnos. Esta parte del proceso enseñanza-aprendizaje contribuye a estimular a los alumnos, incidiendo en su motivación y en la posibilidad de querer aprender más, aspecto que Díaz-Barriga y Hernández (2010) llaman motivación por el aprendizaje.

En este trabajo se propone aplicar el modelo constructivista al diseño de una secuencia didáctica que ayude a los alumnos que cursan Electromagnetismo a transitar gradualmente de su modelo mental a modelos conceptuales del campo eléctrico, induciendo la necesidad de utilizar nuevos conceptos que puedan motivarlos por aprenderlos significativamente.

Para instrumentar la propuesta se utilizará el modelo instruccional de Dick y Carey (1978), ya que es uno de los más completos y flexibles en el ámbito educativo que consiste en nueve pasos muy adecuados de aplicarse en las ciencias con posibilidad de evaluar el avance y con retorno a etapas anteriores. Nuestra propuesta se efectuará al desarrollar las primeras seis etapas de dicho modelo esquematizadas en la Figura 1. 
Figura 1.

Primeras etapas del modelo instruccional

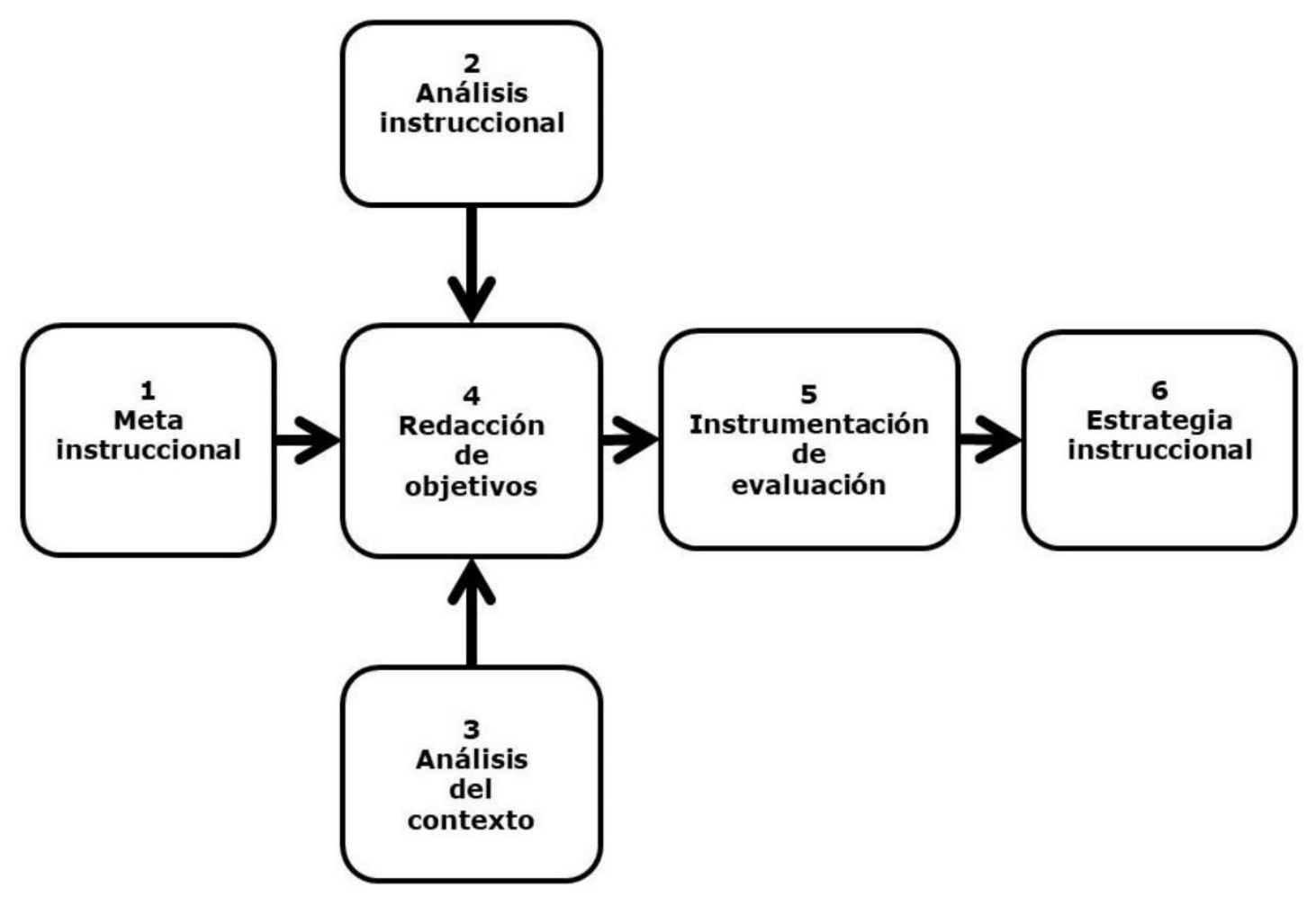

Fuente: Dick y Carey, 1978.

\section{Propuesta didáctica para la enseñanza del campo eléctrico}

El punto de partida de la propuesta es la evaluación diagnóstica (Alonso, 1996). Considerando que el nivel de conocimientos que los alumnos procedentes del bachillerato deben tener, se propone una prueba basada en temas matemáticos y conceptos físicos necesarios para cursar la asignatura de Electromagnetismo. La intención de aplicar un diagnóstico inicial es identificar cuál es el nivel real de conocimientos que los alumnos tienen tanto en aritmética, álgebra, trigonometría y álgebra vectorial, así como en conceptos previos de física, en particular de Electromagnetismo. Algunas de las preguntas de la prueba propuesta se muestran en la Tabla 2.

\section{Tabla 2.}

Ejemplos de preguntas del cuestionario propuesto para identificar el nivel de conocimientos de los alumnos.

Categoría: Aritmética, álgebra y trigonometría.

Instrucciones: Diga si las siguientes afirmaciones son verdaderas o falsas colocando una $V$ o una $F$ en el paréntesis. Si no sabe la respuesta indíquelo marcando la opción $N S$.

1. $\operatorname{Si} \frac{1}{C}=\frac{1}{C_{1}}+\frac{1}{C_{2}}$, entonces $C=C_{1}+C_{2}$. 
5. Si el lado corto de un triángulo rectángulo de $30^{\circ}$ es $1 \mathrm{~cm}$, entonces la hipotenusa es $\sqrt{3} \mathrm{~cm}$.

6. 6 dividido por $\frac{1}{2}$ es 3 .

\section{Tabla 2.}

Ejemplos de preguntas del cuestionario propuesto para identificar el nivel de conocimientos de los alumnos (continuación)

Categoría: Álgebra vectorial y física.

Instrucciones: Elija la opción que considere la respuesta correcta.

12. Si $\vec{B}=(1,2,3)$ y $\vec{C}=(2,1,4)$, ¿cuál de las siguientes operaciones es incorrecta?
(a) $5 \vec{B}$
$=(5,10,15)$
(b) $\vec{B} \cdot \vec{C}=16$
(c) $\quad \vec{C} \times \vec{B}$
$=(5,2,-3)$
(d) $\begin{aligned} & \frac{\vec{B}}{\vec{C}} \\ & =\left(\frac{1}{2}, 2, \frac{3}{4}\right)\end{aligned}$
(e) $\begin{array}{ll}\text { No } & \text { se } \\ \text { entiende la }\end{array}$ pregunta

15. ¿Cuál de los siguientes conceptos físicos se puede representar con un vector?
(a) Trabajo
(b) Temperatura
(c) Calor
(d) Velocidad
(e) entiende la

\section{Fuente: Orear (1980)}

Al mismo tiempo, se debe solicitar a los alumnos que elaboren un mapa conceptual inicial sobre lo relacionado con el campo eléctrico. Es posible que haya alumnos que no conozcan o recuerden cómo elaborarlos, pero el docente podría emplear por lo menos una sesión para aclarar cómo pretende que ellos elaboren su propio mapa conceptual proporcionando los términos más importantes y establecer así las relaciones que consideren adecuadas. En la Figura 2 y Figura 3 se muestran un par de ejemplos de estos mapas conceptuales. 


\section{Figura 2.}

Ejemplos de mapas conceptuales relacionados con antecedentes necesarios para aprender el concepto de campo eléctrico.

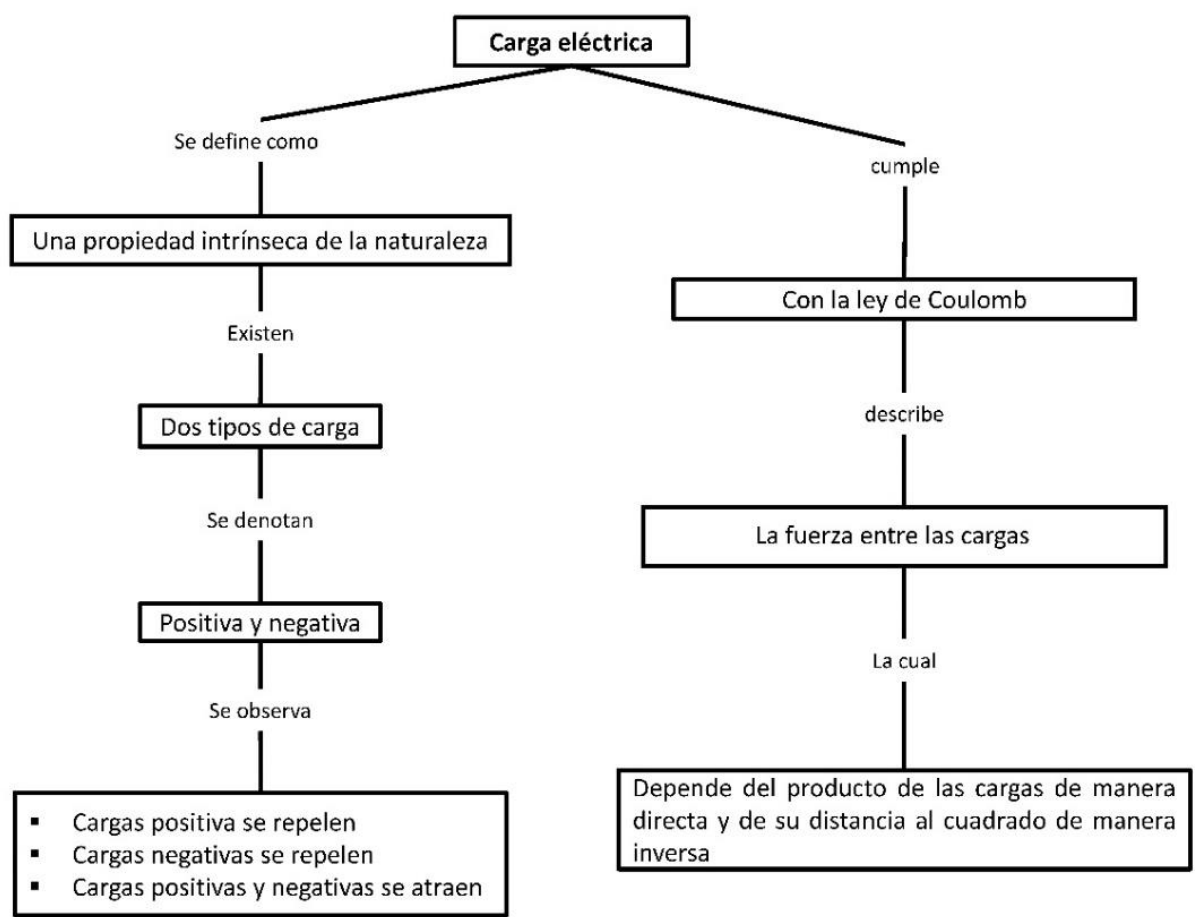

\section{Figura 3}

Ejemplos de mapas conceptuales relacionados con antecedentes necesarios para aprender el concepto de campo eléctrico.

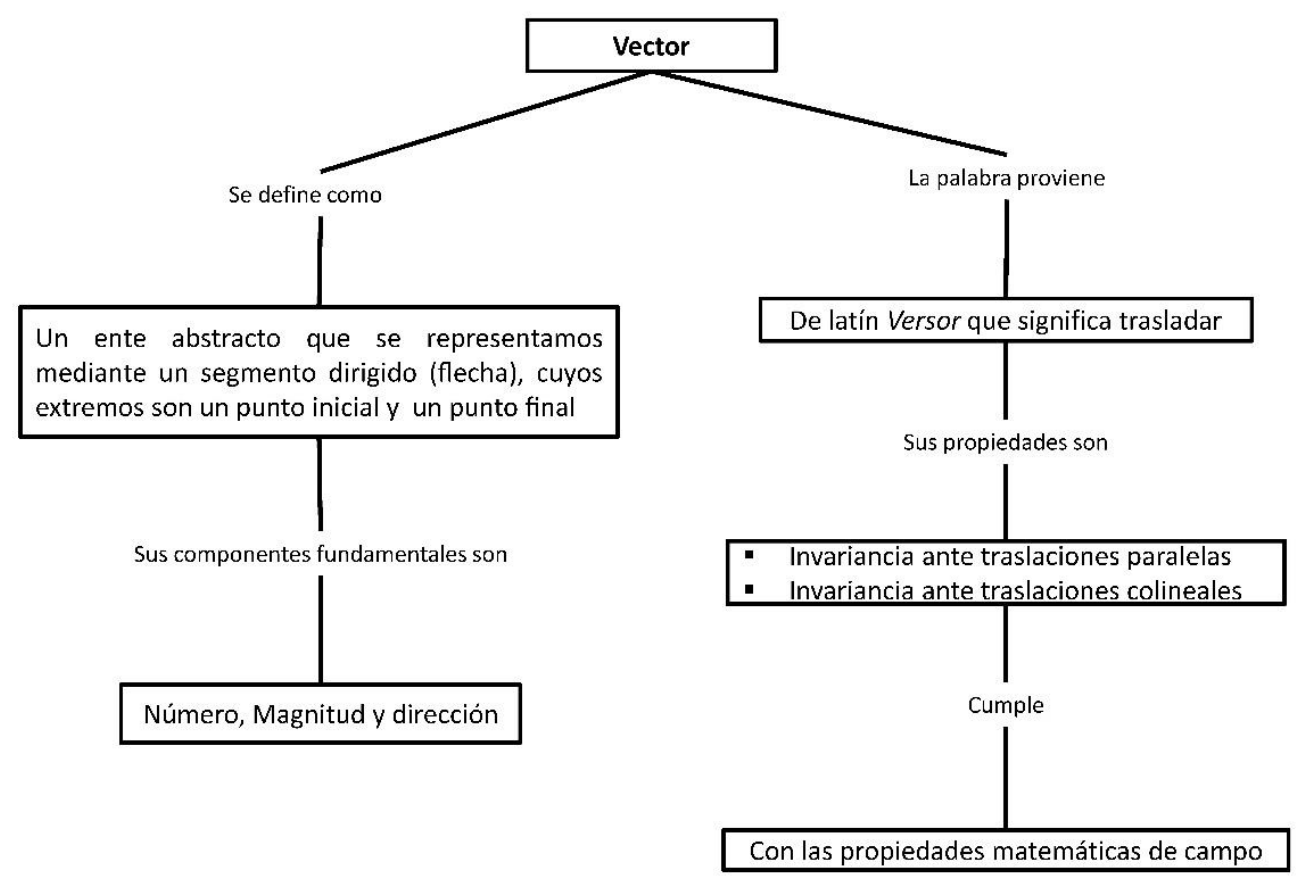

Los resultados obtenidos de la evaluación diagnóstica se pueden agrupar en cuatro clases: los que tienen un conocimiento adecuado de matemáticas y ciertas nociones de física; los 
que tienen un conocimiento adecuado en física, pero no de matemáticas; los que tienen un conocimiento adecuado en matemáticas, pero no en física; finalmente, el grupo de alumnos que presenta muchas deficiencias tanto en matemáticas como en física.

Los alumnos que cuentan con nociones tanto de matemáticas como de física pueden iniciar la instrucción de acuerdo con la secuencia que se propone más adelante (Figura 5). En cambio, aquellos alumnos que tengan alguna problemática con los conceptos y herramientas matemáticas, o con los conceptos previos de la física, será necesario asignarles la realización de lecturas y ejercicios relacionados con la parte específica del tema para que a la brevedad se actualicen y puedan atender la secuencia en igualdad de condiciones.

En el caso de que la mayoría de los alumnos no tenga las nociones mínimas de matemáticas y de física, es muy probable que la instrucción no produzca resultados de aprendizaje de acuerdo a la meta (salvo alumnos sobresalientes en su aprendizaje), porque sería necesario incluir sesiones especiales de regularización a todo el grupo y, por supuesto, reducir la profundidad de los temas por tratar, lo cual afectaría entre otros aspectos la calidad académica de todo el grupo y la eficacia de la enseñanza.

El diseño de estos instrumentos de evaluación, de acuerdo con el enfoque constructivista, es tan importante como la misma secuencia de instrucción, pues funciona como un instrumento de mejora de la enseñanza y por sí mismo del aprendizaje en los alumnos. Se eligen inicialmente los mapas conceptuales y las pruebas escritas con preguntas abiertas de carácter conceptual para evaluar la comprensión de estos.

La propuesta didáctica integra el diagnóstico de los conocimientos previos de los alumnos con los contenidos en forma lógica y secuencial desde el punto de vista del Electromagnetismo. Se considera también necesario que los alumnos realicen una parte experimental tanto para relacionar vivencias reales con sus conocimientos previos como para la estimulación intelectual que pueda influir en su motivación interna. Todos estos aspectos integrados de la propuesta podrán generar una situación propicia de aprendizaje. En la propuesta, estas experiencias y su vinculación con los conocimientos previos en los alumnos tienen un objetivo doble: primero, activar esos conocimientos; segundo, contribuir a su interés por el aprendizaje del Electromagnetismo y sus aplicaciones subsecuentes, como por ejemplo el potencial eléctrico, el flujo eléctrico y el cálculo del campo eléctrico con diferentes configuraciones de carga. Para elaborar la secuencia se usa una jerarquización de conceptos científicos que se han formado con el paso del tiempo (véase la Figura 4).

\section{Figura 4}

Jerarquización de conceptos.

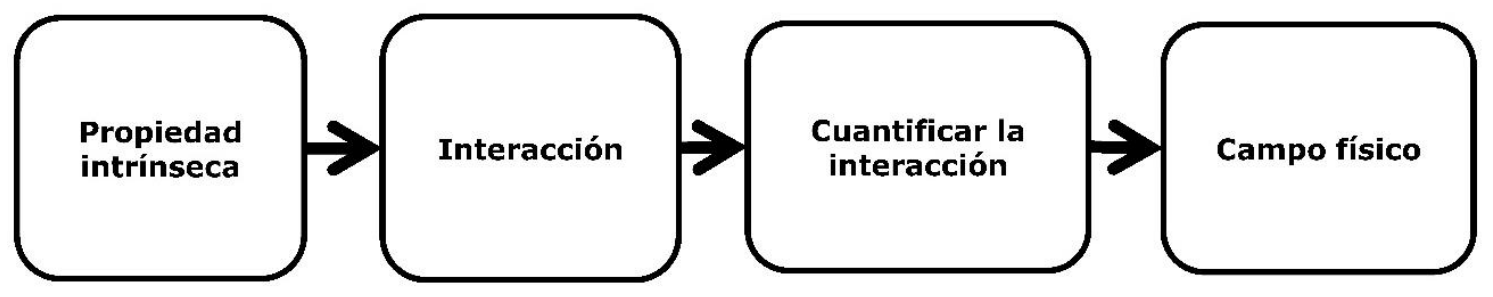


Una adecuación de la presente propuesta a este esquema clásico de enseñanza se inicia con una explicación microscópica de la materia, tratando de que los alumnos establezcan relaciones entre la constitución interna de ésta y sus conocimientos de física y química adquiridos en la preparatoria. Se considera que los alumnos al ser responsables de su aprendizaje y el docente el guía, para que los procesos de aprendizaje estén orientados y produzcan resultados favorables, se intenta desde el inicio realizar conexiones entre los nuevos conceptos y los que el alumno conoce.

La secuencia propuesta se muestra en la Figura 5 e inicia al lograr que el alumno experimente con fenómenos eléctricos de la vida cotidiana, como por ejemplo: frotar un peine con el cabello seco y atraer pedacitos de papel, o frotar con el cabello seco un globo y pegarlo en la pared; generar chispas en la penumbra cuando se separa una cobija de una sábana; experimentar descargas eléctricas cuando se tocan objetos metálicos como el picaporte de una puerta o los carritos de un mercado de autoservicio. Todos estos son ejemplos de fenómenos eléctricos cotidianos que fácilmente se experimentan y repiten comúnmente.

\section{Figura 5}

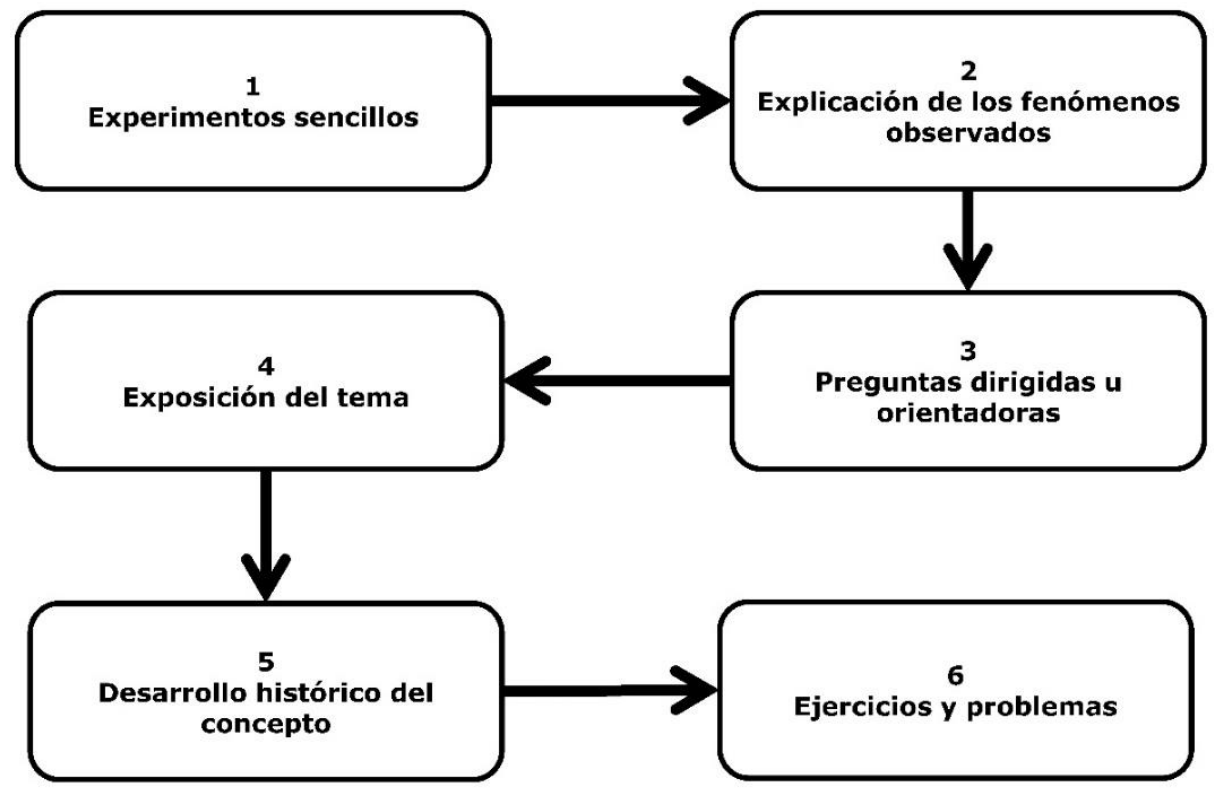

\section{Secuencia Didáctica.}

Una vez introducida esta experiencia sobre los fenómenos eléctricos, el siguiente paso es incrementar el interés del alumno por tales efectos, ensayando con varillas de vidrio y trozos de seda, así como con varillas de plástico y pedazos de piel (que incluya a sus pelos). También pueden utilizarse pequeñas esferas de polietileno y trozos de seda o piel para cargarlas en dos formas diferentes (positiva y negativamente). La intención de estas prácticas es que el alumno elabore conjeturas sobre tales fenómenos, tratando de explicar el origen de la carga eléctrica y las interacciones entre ellas.

Se han considerado estas experiencias por su sencillez, bajo costo y fácil desarrollo en el salón de clase. La siguiente etapa de la secuencia es de reflexión sobre las experiencias eléctricas anteriores y su relación con los conocimientos previos. Los alumnos deben 
comenzar por formular una explicación de los fenómenos efectuados y anotar las observaciones individuales. Estas explicaciones las tienen que entregar al profesor preferentemente después de las prácticas, pues corresponden a una explicación inicial. En caso de no haber tiempo suficiente en ese momento, se sugiere la entrega al siguiente día. Para vincular esta explicación inicial, que parte de los conocimientos previos de los alumnos, con una explicación más profunda de la interacción eléctrica, la instrucción se orientará para que el docente realice preguntas dirigidas como las que se muestran en la Tabla 3.

Tabla 3.

Preguntas orientadoras por parte del docente.

\begin{tabular}{c|c}
\hline \multicolumn{2}{c}{ Preguntas dirigidas } \\
\hline El constructivismo se debate ante tres puntos de vista \\
\hline 1 & ¿Son todas las experiencias eléctricas iguales o existen varios tipos? \\
\hline 2 & ¿Estas experiencias provienen de la masa o de la carga? \\
\hline 3 & ¿La carga eléctrica se conserva o no existe forma de comprobarlo? \\
\hline
\end{tabular}

Con las preguntas dirigidas se intenta guiar al alumno para que comience a preguntarse si existen varios tipos de carga o sólo una — como en el caso de la masa-, establecer una analogía entre la carga y la masa, o plantearse la posibilidad de que la carga pueda conservarse tal como sucede con la masa. La Tabla 4 muestra otras preguntas orientadoras para el aprendizaje del campo eléctrico con la respectiva explicación del porqué de ellas.

\section{Tabla 4.}

Preguntas para favorecer el aprendizaje del campo eléctrico.

\begin{tabular}{ll}
\hline \multicolumn{1}{c}{ Preguntas orientadoras } & \multicolumn{1}{c}{ Para qué } \\
\hline ¿Los fenómenos eléctricos son diferentes & Esta pregunta intenta llevar al estudiante a \\
a los magnéticos o son del mismo tipo? & $\begin{array}{l}\text { la reflexión sobre la posibilidad de que los } \\
\text { fenómenos eléctricos y magnéticos sean la } \\
\text { manifestación de un solo fenómeno, el } \\
\text { electromagnético. }\end{array}$ \\
\hline ¿Por qué en la naturaleza no predominan & Esta pregunta intenta orientar hacia una \\
los fenómenos eléctricos? & respuesta basada en los aspectos reales de \\
& los fenómenos eléctricos; más adelante, se \\
& les mencionará que la interacción eléctrica \\
& es importante y la responsable de la \\
& estructura de la materia a nivel \\
& macroscópico. \\
\hline ¿Se pueden medir los efectos eléctricos? & Esta pregunta intenta que el estudiante \\
& piense en cómo medir los efectos eléctricos \\
& y se dé cuenta que la cuantificación no es \\
& asunto simple, motivo por el cual \\
& transcurrieron muchos años desde que se \\
& observaron los primeros efectos hasta la \\
& cuantificación de estos. \\
\hline
\end{tabular}


Es posible efectuar otras preguntas según el criterio y profundidad que cada docente considere oportuno. Se trata de activar los conocimientos previos y estimular el interés de los alumnos por aprender más conceptos relacionados con la interacción eléctrica. Antes de comenzar la forma expositiva de esta secuencia, se solicitará a los alumnos que presenten un ensayo individual -el cual debe ser revisado-, donde indiquen sus explicaciones y, si fuera posible, las nuevas ideas que hayan adquirido permitiéndoles la comprensión de nuevos conceptos.

La exposición de la teoría comienza por una explicación simplificada de la constitución interna de la materia, hablando de átomos y de sus componentes tales como los electrones, protones y neutrones. Con ello se hará recordar al alumno que dichos componentes tienen masa y un tipo de carga, si son protones carga positiva o si son electrones carga negativa. Ambas propiedades intrínsecas de la materia se pueden extrapolar al mundo macroscópico y convertirse en las propiedades clásicas que conocemos: la masa y la carga.

Hay que ser precavidos al explicar a la materia y sus propiedades de esta forma, porque en el afán de clarificar los conceptos en el mundo macroscópico podrían surgir más preguntas de los alumnos interesados en indagar si la teoría clásica del Electromagnetismo se aplica a este nivel atómico. Se recomienda a los profesores indicar al alumno interesado que las teorías para ambos niveles son diferentes. Por ejemplo, en el caso microscópico corresponde al ámbito de la física moderna, asignatura que se estudia en cursos especializados con referencias específicas. La teoría del Electromagnetismo que se enseñará en esta secuencia, y que está especificada en los programas oficiales, corresponde al ámbito de la física clásica.

La razón de recurrir a una visión microscópica de la materia, dentro de la teoría clásica del Electromagnetismo, es que varias de las interacciones en lo cotidiano tienen que ver con esta clase de fenómenos microscópicos que se pueden explicar satisfactoriamente.

Típicamente, la estructura ordenada en forma de cristales que los sólidos poseen se explica con base en las redes moleculares y en los enlaces que tienen los átomos. Muchas características de los sólidos como conductores, aislantes o semiconductores se pueden explicar también con la teoría atómica. Es posible indicar en alguna etapa de la exposición que la carga eléctrica (positiva o negativa) puede visualizarse como el déficit o exceso de electrones, pues estas partículas son las que tienen la movilidad para contribuir a las propiedades globales de la materia.

Los alumnos pueden tener explicaciones incompletas o alternativas de las que se considera la teoría científica, pero es necesario que las puedan externar de forma escrita u oral. Incluso, el no saber, representado comúnmente por la frase no lo sé, podría indicar que el alumno no está interesado en expresar su respuesta. También se solicitará a los alumnos que preparen una serie de explicaciones sobre conceptos tales como materia, carga e interacción eléctrica presentando un ensayo propio donde señalen el resultado de sus reflexiones. Éste puede y debe complementarse con diversas fuentes de consulta (sitios Web o textos clásicos). Esta etapa prepara el escenario para que el docente continúe su exposición hablando de la carga y la interacción eléctrica. 
La explicación continúa con las propiedades de la carga eléctrica (los tipos de carga), el principio de conservación de la carga como una propiedad comprobable experimentalmente (sin excepción alguna), así como la ley de las cargas que muestra experimentalmente que si son del mismo o diferente grupo se rechazan o atraen, respectivamente.

Después se hablará de la relación entre cargas puntuales y la manera en que se obtuvo la ecuación de Coulomb. Se mencionará específicamente el experimento clave para cuantificar la interacción eléctrica tratando de relacionar el concepto de fuerza, visto en cursos anteriores de física, con el de fuerza eléctrica como ejemplo sobresaliente de lo conocido como interacción eléctrica.

A continuación, se aproxima la etapa de evaluar si el alumno ha comprendido en alguna forma los conceptos de materia, carga e interacción eléctrica tal que pueda establecer relaciones significativas con los conocimientos previos que conoce. Suponiendo que el alumno tiene claridad, estructura, secuencia lógica y efectúa algunas reflexiones, estamos en posibilidad de considerar si la explicación científica de la carga y la interacción eléctrica ha sido importante para ellos.

En esta parte de la instrucción es donde la propuesta tiene su aspecto medular, es decir, se pretende inducir a los alumnos a que efectúen un razonamiento tal que sientan la necesidad de modificar sus conocimientos, bajo el logro de aprendizaje significativo, lo más apegado a los modelos científicos que el profesor expone mediante esta secuencia. Desafortunadamente, no se conoce una manera eficaz de comprobar si el alumno ha llegado a una desestabilización cognitiva - comentado anteriormente con la teoría de Piaget- o sólo está participando pasivamente en la instrucción.

La confrontación de ideas y conceptos es necesaria para que el alumno pueda comprender y asimilar los nuevos conceptos, aunque el tiempo que tome en modificar sus esquemas anteriores y estructura de pensamiento, no necesariamente sigue el programa del calendario escolar, es decir, el tiempo para alcanzar la comprensión difiere entre un estudiante y otro. No obstante, hace falta una aplicación concreta de los conceptos, considerados abstractos, porque a medida que se avanza en el desarrollo de la teoría resultan más distantes de lo cotidiano, así como en su aplicación directa.

Por lo anterior, una sugerencia es efectuar una prueba intermedia donde se respondan preguntas conceptuales con aplicación de la teoría, seleccionadas de los textos clásicos y elaboradas por el profesor, de acuerdo con la relación concepto-aplicación en situaciones típicas que se quiera evaluar. Esta evaluación no sólo permitirá detectar el avance en la instrucción, sino también ayudará a que los conceptos no queden aislados de manera que el alumno perciba que existe relación entre todos ellos y que se requiere aprenderlos con mayor hondura.

Antes de pasar a las explicaciones del campo eléctrico, es necesario indicarle al alumno la posibilidad de ampliar la comprensión de la interacción eléctrica considerándolo una herramienta conceptual más poderosa.

El siguiente apartado de la secuencia didáctica también es mediante técnica expositiva, donde se promueve la comprensión de la ciencia a través de su evolución histórica y de las preguntas que se hacían los científicos en sus primeras investigaciones. De tal manera que lo que actualmente se conoce como campo eléctrico, surgió de la necesidad de crear 
el concepto de campo al tratar de explicar la acción a distancia de varias interacciones de la naturaleza. Una de las mejores aproximaciones fue la de usar las "líneas de fuerza" de Faraday (Berkson, 1993); que, aunque en la actualidad estas líneas de fuerza se consideran imaginarias, ayudan a visualizar los efectos de un campo eléctrico que sí es real. De hecho, Faraday utilizó dichas líneas de fuerza porque no tenía gran instrucción matemática, aun cuando tenía claro el concepto de fuerza (concepto de la mecánica clásica). Nuestra propuesta considera que es más satisfactoria la explicación de las líneas de fuerza antes de comenzar la explicación del campo eléctrico.

En este sentido, se solicitará al alumno su opinión por escrito acerca de la acción a distancia y cómo percibe las interacciones de tipo gravitacional o eléctrica cuando la distancia entre masas o cargas puntuales son suficientemente grandes. Con base en esta percepción y en los conceptos aprendidos por el estudiante, el docente explicará la necesidad de que los objetos interaccionen a través de un campo tomando en cuenta el efecto del tiempo. Para decirlo de una manera sencilla, el campo existe debido a la propiedad que se trate de medir y la comprobación surge en la medición de sus efectos, es decir, la interacción sólo se produce con la misma propiedad entre dos objetos, masas o cargas, por ejemplo.

La secuencia continúa con la aplicación del concepto de campo eléctrico a la resolución de varios ejemplos clásicos: la configuración de una carga positiva en interacción con una negativa, dos cargas positivas o negativas, el dipolo y distribuciones lineales o circulares de carga. En esta propuesta no se recomienda resolver ejercicios con una distribución volumétrica de carga por la dificultad matemática que probablemente no ayude a la comprensión del tema, sino que por el contrario inhiba su aprendizaje. De acuerdo con el nivel de su grupo, el docente decidirá y propondrá a la clase la realización de ejercicios variados con diferentes distribuciones de carga que ayuden a reforzar el concepto del campo eléctrico y su aplicación.

Se recomienda aplicar un cuestionario específico siguiendo un modelo de preguntas abiertas para indagar en la comprensión de los conceptos de carga, interacción y campo eléctrico, ver Tabla 5. También puede solicitar a los alumnos la elaboración de un mapa conceptual sobre los contenidos aprendidos. Ambas herramientas de evaluación se cotejan con las del diagnóstico inicial pudiendo así efectuar un balance sobre los resultados alcanzados.

\section{Tabla 5.}

Cuestionario sobre la comprensión de conceptos.

\begin{tabular}{l|l}
\hline \multicolumn{2}{l}{ Cuestionario sobre comprensión de conceptos } \\
\hline 1 & $\begin{array}{l}\text { Si los electrones tuvieran carga positiva y los protones carga negativa, ¿serían } \\
\text { distintas las interacciones físicas y químicas? }\end{array}$ \\
\hline 2 & $\begin{array}{l}\text { Si cargamos un peine o una varilla de plástico frotándolo con un paño de } \\
\text { seda, ¿puede determinar si adquiere carga positiva o negativa? }\end{array}$ \\
\hline 3 & $\begin{array}{l}\text { ¿Cuál es la dirección y sentido del campo eléctrico con respecto a la fuerza } \\
\text { eléctrica? }\end{array}$ \\
\hline 4 & $\begin{array}{l}\text { De forma cotidiana no percibimos las fuerzas eléctricas entre dos objetos } \\
\text { cualesquiera, ¿cuál es a razón de esto? }\end{array}$ \\
\hline
\end{tabular}




\begin{tabular}{l|l}
\hline 5 & Describa cuál era el objetivo del experimento de la gota de aceite de Millikan. \\
\hline 6 & $\begin{array}{l}\text { Explique por qué las cargas que se utilizan para medir el campo eléctrico } \\
\text { deben ser muy pequeñas. }\end{array}$ \\
\hline 7 & $\begin{array}{l}\text { ¿Qué son las líneas de fuerza eléctrica? ¿Hablar de líneas de fuerza eléctrica } \\
\text { es equivalente a hablar de líneas de campo eléctrico? }\end{array}$ \\
\hline 8 & ¿Se pueden cruzar las líneas de campo eléctrico? \\
\hline 9 & ¿El campo eléctrico existe en el vacío? \\
\hline 10 & Si se carga eléctricamente una pompa de jabón, ¿qué pasa con su tamaño? \\
\hline
\end{tabular}

\section{CONCLUSIONES}

Existe una problemática de enseñanza y aprendizaje de los conceptos básicos del Electromagnetismo: la carga, la interacción eléctrica, la corriente y los campos eléctrico y magnético. Estos son conceptos abstractos y de difícil comprensión al dificultarse una asociación por analogía. El presente trabajo pretende coadyuvar a resolver este problema mediante una propuesta didáctica tal que el estudiante aprenda significativamente el concepto de campo eléctrico. Para lograr dicho objetivo se proponen secuencias didácticas que deberán ser ajustadas a las características de cada grupo, principalmente con base en los resultados del examen diagnóstico y las posibilidades para obtener materiales e información del docente y sus estudiantes.

Consideramos que la falta de comprensión de los conceptos, por razones comentadas, es uno de los factores que desmotiva a los estudiantes a involucrase en la materia de estudio, y a la vez conduce al recurso memorístico de los contenidos para solventar un compromiso (tarea, examen, entre otros). Es evidente que las ejecuciones de este estilo por parte del aprendiz son insuficientes respecto de la dimensión de métodos y significados de la asignatura y que es una barrera conceptual en la aplicabilidad de otros ámbitos de estudio o trabajo futuro.

En este trabajo, con base en el enfoque constructivista del aprendizaje, se ha presentado una didáctica centrada en el alumno considerándolo un sujeto, con conocimientos previos, capaz de elaborar por sí mismo su proceso de aprendizaje tal que el docente funge como figura responsable de orientar los procesos y las estrategias pertinentes.

La propuesta aquí desarrollada, representa el esfuerzo por considerar a los alumnos en un compromiso de participación en los procesos de enseñanza, donde el docente necesita pensar en estrategias para involucrarlos, y por ende en los de su aprendizaje. Si bien la postura de la propuesta es didáctica, no puede ignorarse el objetivo principal de que la enseñanza escolar debe promover un mejor aprendizaje en los alumnos, enfocado al promedio de los alumnos que llegan a las aulas de nivel superior.

Finalmente podemos comentar que estamos conscientes que la aplicación de esta propuesta depende de las características de los estudiantes, por lo que el diseño exitoso del docente en un curso o semestre no garantiza que así lo será si sólo lo reproduce de manera idéntica en cada vez que imparta la asignatura, sino que siempre deberá partir del grupo que reciba. 


\section{REFERENCIAS BIBLIOGRÁFICAS}

Alonso, M. (1996). La evaluación en la enseñanza de la Física como instrumento de aprendizaje (Tesis doctoral). Universidad de Valencia.

Berkson, W. (1993). Las teorías de los campos de fuerza. Desde Faraday hasta Einstein. Alianza Editorial.

Campelo, R. (2003). Un modelo didáctico para enseñanza aprendizaje de la física. Revista Brasileira de Ensino de Física, 25(1), 86-104.

Carretero, M. (2009). Constructivismo y educación. Paidós.

Coll, C. (2001). Constructivismo y educación. La concepción constructivista de la enseñanza y el aprendizaje. En C. Coll, J. Palacios y A. Marchesi (Eds.), Desarrollo psicológico y educación 2: Psicología de la Educación escolar (pp. 153-178). Alianza.

Díaz-Barriga, F. y Hernández, G. (2010). Estrategias docentes para un aprendizaje significativo: una interpretación constructivista. McGraw Hill.

Dick, W. \& Carey, L. (1978). The systematic design of instruction. Scott, Foresman \& company.

Flórez, R. (2005). Hacia una Pedagogía del conocimiento (2 ${ }^{\mathrm{a}}$ Ed.). McGraw-Hill.

Hernández, P. (2000). Diseñar y enseñar: Teoría y técnica de la programación del proyecto docente. Narcea.

Jiménez, A. (2011). Análisis de índices de reprobación registrados en el Instituto Tecnológico de Querétaro en el período 2009-2010. Crónica Naranja, 6(25), 4143.

Llancaqueo, A. (2006). El aprendizaje del concepto de campo en Física: conceptualización, progresividad y dominio. (Tesis doctoral). Universidad de Burgos.

Mayorga, J. y Madrid, D. (2010). Modelos didácticos y Estrategias de enseñanza en el Espacio Europeo de Educación Superior. Tendencias pedagógicas, 15(1), 91111.

Mora, C. y Sandoval, M. (2009). Modelos erróneos sobre la comprensión del campo eléctrico en estudiantes universitarios. Latin-American Journal of Physics Education, 3(3), 647-655.

Moreira, M. (1997). Aprendizaje significativo: Un concepto subyacente. Actas del Encuentro Internacional sobre Aprendizaje Significativo (pp. 19-44). Universidad de Burgos.

Moreira, M., Greca, I. y Palmero, L. (2002). Modelos mentales y modelos conceptuales en la enseñanza y aprendizaje de las ciencias. XX Encuentros didácticos de la ciencia experimental en La laguna, Tenerife. Universidad de La Laguna.

Orear, J. (1980). Física Fundamental. Limusa.

Pozo, I. (1997). ¿Por qué los alumnos no aprenden la ciencia que les enseñamos? El caso de las ciencias de la tierra. Enseñanza de las Ciencias, 8(1), 3-19.

Rivera, J. L. (2004) El Aprendizaje Significativo. Revista de Investigación Educativa, $8(14), 47-52$. 
Ruiz, F. (2007). Modelos didácticos para la enseñanza de las ciencias naturales. Revista latinoamericana de estudios educativos, 3(2), 41-60.

Saldarriaga-Zambrano P., Bravo-Cedeño, G. y Loor-Rivadeneira, M. (2016). La teoría constructivista de Jean Piaget y su significación para la pedagogía contemporánea. Dominio de las Ciencias, 2 (Núm. Esp.) 127-137.

\section{PARA CITAR EL ARTÍCULO INDEXADO.}

Cerriteño Rodríguez, O., Sánchez Moreno, A., Álvarez López, J. L., \& Aguilera Terrats, J. R. (2021). Propuesta constructivista para la enseñanza del campo eléctrico. Magazine De Las Ciencias: Revista De Investigación E Innovación, 6(1), 27-66. https://doi.org/10.33262/rmc.v6i1.1068 